WÜST, Wolfgang, Reichskreis und Territorium : Die Herrschaft über der Herrschaft? Supraterritoriale Tendenzen in Politik, Kultur, Wirtschaft und Gesellschaft. Ein Vergleich süddeutscher Reichskreise

Christophe Duhamelle

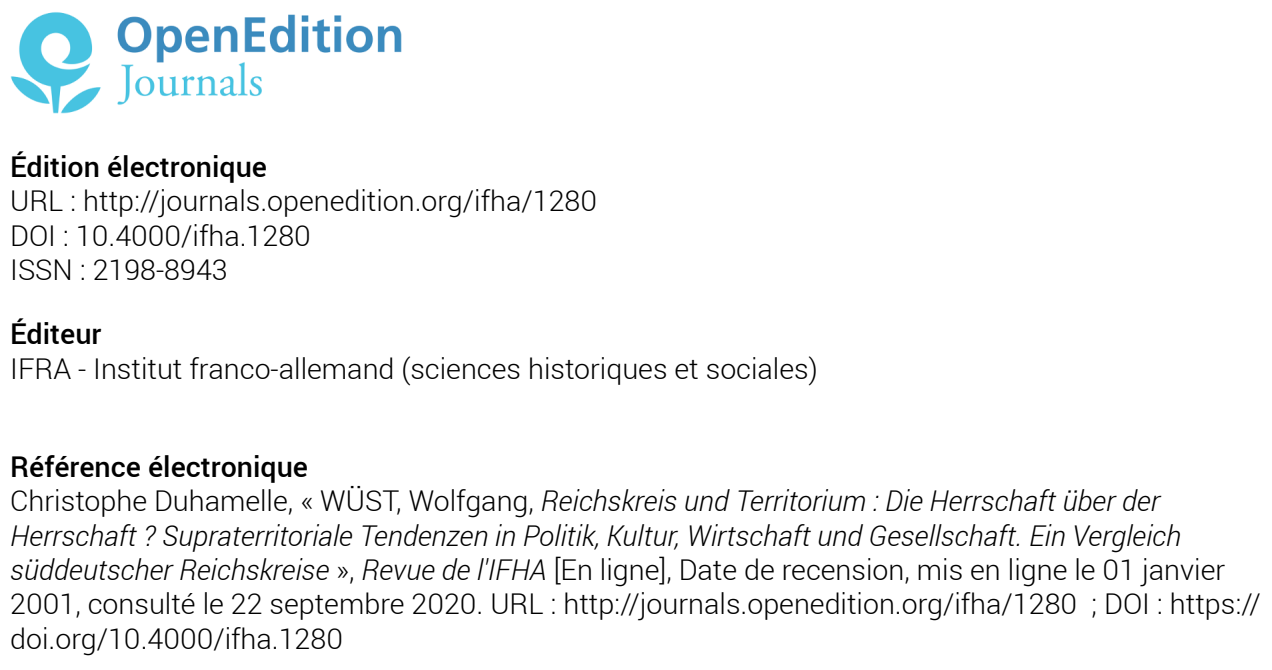

Ce document a été généré automatiquement le 22 septembre 2020.

(CIFHA 


\title{
WÜST, Wolfgang, Reichskreis und Territorium : Die Herrschaft über der Herrschaft ? Supraterritoriale Tendenzen in Politik, Kultur, Wirtschaft und Gesellschaft. Ein Vergleich süddeutscher Reichskreise
}

\author{
Christophe Duhamelle
}

1 Terra incognita de la recherche allemande il y a quinze ans à peine, les Cercles d'Empire ont bénéficié du regain d'intérêt envers les institutions impériales à l'époque moderne mais aussi de l'attention accrue portée à la diversité et à la collégialité introduites par le caractère partagé et disséminé de l'autorité politique dans le SaintEmpire. La volonté de mettre au jour, parfois de manière un peu forcée, les fondements historiques de la démocratie et du fédéralisme allemands joue aussi un rôle dans cette redécouverte. Après le recueil Regionen in der Frühen Neuzeit dirigé par Peter Claus Hartmann (voir BullMHFA, 30/31, 1995, p. 254-255) et le précis de Winfried Dotzauer (voir BullMHFA, 35, 1999, p. 280-281), ces actes d'un colloque de 1998 constituent un ensemble bien composé et illustré venant s'ajouter à une bibliographie dont on peut regretter qu'elle n'ait pas été rassemblée en fin de volume.

2 Ne serait-ce qu'en raison de son morcellement politique qui contraignait beaucoup de territoires à choisir entre l'insignifiance politique ou la coopération supra-territoriale, ainsi que de la proximité plus grande de l'empereur et des institutions impériales, c'est le Sud-Ouest de l'Empire qui abritait les Cercles les plus actifs, et donc les plus étudiés. Cet ouvrage n'échappe pas à la règle. Après avoir proposé cinq réflexions générales (P.C. HARTMANN sur les fonctions des Cercles, E.J. WEBER sur le faible intérêt qu'ils suscitent au sein de la publicistique impériale, H. NEUHAUS sur leur rôle dans les guerres d'Empire et la pépinière d'officiers qu'ont constituée les armées des Cercles 
après la réforme de 1681-82, M. PLASSMANN sur les performances militaires, efficaces dans un cadre défensif, des Cercles occidentaux et B. WUNDER à propos de l'édit impérial sur l'émigration qui, en 1768, montre l'influence politique conservée par les Cercles), le recueil comprend en effet, sur les treize articles restant, huit études portant sur la Souabe et la Franconie. Ces contributions approfondissent nos connaissances sur l'action des Cercles en matière de maintien de l'ordre (en particulier dans la lutte contre le vagabondage, thème sur lequel les territoires reprennent souvent, en Souabe, les délibérations des assemblées de Cercle, $\mathrm{W}$. WÜST), de régulation économique (R. ENDRES sur la Franconie, où une ordonnance de police du Cercle a été promulguée en 1572, et où le Cercle ne cesse d'agir en faveur d'une unification des douanes et de l'approvisionnement céréalier) et de politique envers les Juifs (S. ULLMANN note une évolution vers le pragmatisme et, malgré une gestion avant tout territoriale, une réflexion commune du Cercle de Souabe sur les Juifs marginaux). Le fonctionnement interne des Cercles fait également l'objet d'une attention accrue : A. SCHMID fait ainsi le bilan des équilibres au sein du Cercle de Franconie, qui a connu 322 réunions générales, tandis que B. SICKEN en montre la constante évolution institutionnelle, qui résiste même au passage des margraviats de Bayreuth et Ansbach à la couronne de Prusse en 1791. Ulm, « capitale » du cercle de Souabe, reçoit de la présence des délégués de chaque territoire une impulsion économique et culturelle qui se reflète en particulier dans la vitalité du théâtre (H.E. SPECKER). Ces institutions « fédérales » ont donc laissé de nombreuses traces archivistiques, que leur dispersion ultérieure et leur répartition dans les dépôts de chaque territoire rendent malheureusement difficiles d'accès (B. THEIL, R. HEYDENREUTER).

3 Les autres Cercles sont moins connus, car ils ne jouaient qu'un rôle limité : ce sont les grandes principautés territoriales qui mènent le jeu, à l'instar de la Bavière, dont la politique dans le Cercle de Souabe s'effectue plus en fonction de visées dynastiques que selon les idéaux d'égalité du Cercle (W. LIEBHART) mais qui pourtant laisse subsister, dans le Cercle même de Bavière, les institutions et les terrains d'action de la coopération supra-territoriale (P.C. HARTMANN). Cette survie marginale, qui caractérise aussi le Cercle d'Autriche (où les Habsbourg trouvent une échelle intermédiaire commode pour leurs relations avec le reste de l'Empire, A.K. MALLY) et renaît parfois à la faveur de dangers extérieurs (ainsi que le montre l'association des deux Cercles saxons et du Cercle de Franconie contre Louis XIV, T. NIKLAS), peut cependant déboucher sur un inattendu regain de vigueur au XVIIIe s., comme c'est le cas dans le Cercle de Haute-Rhénanie après que les conflits confessionnels, conversions princières aidant, se sont apaisés (K. AMANN).

4 Un riche recueil, donc. On ne peut cependant que partager le souhait, exprimé en introduction, d'un élargissement de l'étude des institutions impériales vers leur dimension sociale, culturelle et de "culture politique ", tendance illustrée d'ailleurs par quelques articles de ce livre. 